\title{
Glycaemic control, glucose variability and the Triangle of Diabetes Care
}

\author{
GERRY RAYMAN
}

\begin{abstract}
The discovery of insulin turned a diagnosis of type 1 diabetes from a terminal condition to one that can be managed in a way that allows a full and fulfilling life. Optimal management of glycaemia plays a key role within the long-term management of diabetes. Indeed, the Diabetes Control and Complications Trial and the UK Prospective Diabetes Study established beyond doubt that intensive management of blood glucose $\left(\mathrm{HbA}_{1 \mathrm{c}}\right)$ reduced the risk of long-term (especially microvascular) complications of the disease in type 1 and type 2 diabetes, respectively. Long-term observational follow up years or decades beyond these trials revealed a longer-term macrovascular benefit from these interventions. There is more to glycaemic control than the prevailing level of $\mathrm{HbA}_{1 \mathrm{c}}$, however. Variability of blood glucose within and between days promotes hypoglycaemic and hyperglycaemic episodes that may increase the risk of diabetes complications or adverse clinical outcomes and which certainly impair patients' quality of life and confidence in managing their insulin regimen. The Triangle of Diabetes Care has emerged as a useful concept here, bringing together the need to improve glucose levels, but also to avoid hypoglycaemia and to reduce glucose variability. Continuous glucose monitoring is a particularly valuable tool for addressing glycaemic variability, but patients and healthcare professionals can be swamped by the large amount of data that it generates. Advanced glucose profiling provides a means of producing a straightforward, visual representation of daily glucose profiles over a number of days that can help to pinpoint the changes in the insulin regimen needed to optimise blood glucose control.

Br J Diabetes 2016;16(Suppl1):S3-S6
\end{abstract}

Key words: Glucose monitoring, glucose variability, glycosylated haemoglobin, diabetes complications, hypoglycaemia

Diabetes Foot Clinic and Diabetes Research Unit, Ipswich Hospital, Suffolk, UK

Address for correspondence: Gerry Rayman

The Diabetes Centre and Diabetes Research Unit, Ipswich Hospital, Suffolk, IP4 5PD, UK

E-mail: Gerry.Rayman@ipswichhospital.nhs.uk

http://dx.doi.org/10.15277/bjd.2016.070
Introduction

Prior to the discovery of insulin, type 1 diabetes was a fatal illness, and death often occurred within days or weeks of diagnosis. In 1919, Elizabeth Hughes developed "juvenile" (type 1) diabetes at age 11 years and barely survived on the starvation diet that was the only therapeutic option of the time. ${ }^{1,2}$ Banting himself examined her in 1922, at which time she was weak and extremely emaciated, weighing only $20 \mathrm{~kg}$. Rescued from certain death by insulin, she went on to write "To think I'll be leading a normal, healthy existence is beyond all comprehension...Oh, it is too wonderful for words this stuff". "

Nevertheless, this impure extract of insulin was beset with many problems, of which hypoglycaemic and hyperglycaemic coma were the most concerning. Therapy was therefore aimed at maintaining a high enough blood glucose level to avoid hypoglycaemia but not high enough to tip patients into diabetic ketoacidosis (DKA). Insulin preparations with longer durations of action were devised to achieve this, avoiding the need for multiple daily injections. Deaths from DKA fell dramatically; however, years later it was realised that insulin was not quite the 'cure' hoped for, as people with type 1 diabetes now survived to develop long-term complications which were previously uncommon. Blindness, death from renal failure and amputation now became the feared consequences of having diabetes. Patients diagnosed with type 1 diabetes in their teens were found to have only a $50 \%$ chance of surviving into their fifties.

Improved glycaemic control reduces the risk of diabetes complications

In the face of growing numbers of patients developing complications, the role of glycaemic control was increasingly debated; however it was not until the late seventies that technological advances made it possible to undertake prospective studies to examine the role of good glycaemic control on prevention of diabetes complications. The identification of $\mathrm{HbA}_{1 c}$ as a measure of long-term glycaemic control in 1975 by Koenig and Cerami was key to unravelling the relationship between glycaemic burden and diabetes complications. ${ }^{3}$ Indeed, $\mathrm{HbA}_{1 \mathrm{c}}$ remains an essential measure by which to assess the quality of care of diabetic patients today. ${ }^{4}$

Home blood glucose monitoring represents another essential development in diabetes care. Studies from the late 1970s showed that home blood glucose monitoring was not only possible but helped patients and health care professionals to more intelligently adjust the insulin dose to improve control. Although this used systems designed for use on wards, these early successes led to the introduction of smaller, more practical self-mon- 
itoring blood glucose (SMBG) meters in the early 1980s. These, together with purified insulin preparations with better defined pharmacokinetics, disposable plastic syringes and later insulin pen devices facilitated the introduction of the basal-bolus regimens that were used in the Diabetes Control and Complications Trial (DCCT). ${ }^{5}$ This landmark trial, published in 1993, demonstrated that more vs. less intensive management of glycaemia $\left(\mathrm{HbA}_{1 \mathrm{c}}\right)$ reduced the risk of long-term diabetes complications. ${ }^{5}$ Five years later, the UK Prospective Diabetes Study (UKPDS) demonstrated comparable microvascular benefits from intensive glycaemic management in people with type 2 diabetes. ${ }^{6}$ Longterm follow-up from these studies suggested that early application of intensive glycaemic management provided long-term macrovascular benefits years - or decades - after the end of the trial, in addition to the microvascular benefits observed during the randomised phases of the trials. 7,8 In this sense, optimal glycaemic control, applied early, is the "gift that keeps on giving ". .9

\section{Limitations of $\mathrm{HbA}_{1 \mathrm{c}}$ and glucose variability}

$\mathrm{HbA}_{1 \mathrm{c}}$ is an average measure of glycaemic control over about the preceding three months, and tells us nothing about the short-term variability of blood glucose. Thus, the level of $\mathrm{HbA}_{1 \mathrm{c}}$ may also mask highly variable and potentially dangerous diabetes control. An apparently 'good result' may be seen in patients who have wild swings from hyperglycaemia to hypoglycaemia, the latter often occurring unnoticed at night. Conversely, overtreatment of frequent hypoglycaemia, or defensive eating to ward off hypoglycaemia, may contribute to persistent hyperglycaemia. ${ }^{10}$ Such variability in blood glucose may significantly impact on the patient's well-being and their satisfaction with their diabetes control. ${ }^{7}$

Although the relationship between $\mathrm{HbA}_{1 \mathrm{c}}$ and the risk of diabetes complications is beyond question, ${ }^{11}$ it has been estimated that total glycaemic exposure (derived from average $\mathrm{HbA}_{1 \mathrm{c}}$ and duration of diabetes) only predicted $11 \%$ of the risk of developing retinopathy in the type 1 diabetic cohort of the DCCT cohort. ${ }^{12}$ Increasing evidence implicates glucose variability as an important contributor to the development of diabetes complications, although this remains controversial. ${ }^{13}$ Nevertheless, it would seem sensible to minimise glucose variability as it is associated with reduced patient satisfaction and increased hypoglycaemia; the latter being shown to trigger inflammatory responses that have been linked to impairment of vascular function. 8,14

\section{Hypoglycaemia}

Socio-economic, cultural and educational barriers may contribute to poor control of diabetes. ${ }^{15}$ However, fear of hypoglycaemia is a major barrier for those with type 1 diabetes striving to achieve good control as well as for people with type 2 diabetes on insulin and/or sulphonylureas. ${ }^{16}$ The impact of hypoglycaemia can range from being a nuisance to being immediately life-threatening; hypoglycaemia can be unpleasant, a cause of embarrassment and disabling; it can promote discrimination at work or school and loss of a driving licence. ${ }^{13}$ Severe hypoglycaemia can cause neurological disability, seizures, coma and injury, and has been associated with increased mortality. ${ }^{13-18}$ Recurrent hypoglycaemia is a particularly vicious problem as it may lead to hypoglycaemia unawareness, with loss of early warning signs of hypoglycaemia and increased risk of further and potentially more profound and dangerous hypoglycaemic episodes. ${ }^{14}$ In the DCCT, intensive vs. standard glycaemic control was associated with a 3.3-fold increase in the rate of severe hypoglycaemia, with a clear association between hypoglycaemia and achieved $\mathrm{HbA}_{1 \mathrm{c}} \cdot{ }^{19}$

\section{Introducing the Triangle of Diabetes Care}

From the above discussion it can be seen that there are three goals in diabetes management. These are, reducing overall glycaemic burden which is at present assessed by $\mathrm{HbA}_{1 \mathrm{c}}$, preventing hypoglycaemia and reducing glucose variability.

Ideally, all three targets should be achieved but this is may not always be possible or necessarily desirable. For example, the target $\mathrm{HbA}_{1 \mathrm{c}}$ may be more stringent in a young relatively newlydiagnosed patient who has many future decades of life with diabetes ahead of them and less stringent in an older, frail individual with cardiovascular disease in whom the clear priority should be avoiding the potential harm associated with hypoglycaemia.

In contrast, reducing glucose variability will be beneficial whatever the $\mathrm{HbA}_{1 \mathrm{c}}$ target, in smoothing peaks and troughs in glucose levels, both of which have been linked to increased mortality. ${ }^{8}$ Variability may exist within and between days of the week. For example, pre-breakfast glucose during the working week may be different from at the weekend due to different times of waking and different events on the evening before.

Figure 1. The Triangle of Diabetes Care

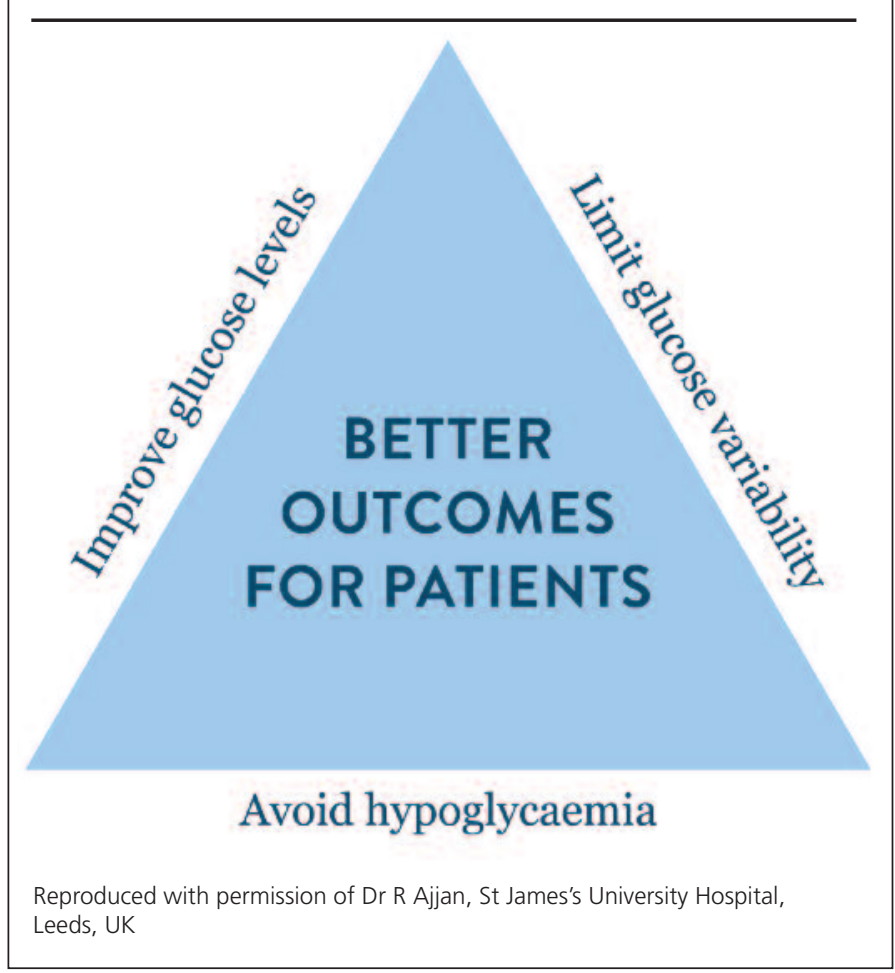


It is important for patients to understand the causes of this variability and how to minimise it, so that it does not become a source of frustration and loss of confidence in insulin therapy and glucose monitoring. Within-day variability can reflect a missed insulin dose or excess carbohydrate intake, or if repeated, may represent an imbalance in insulin treatment; for example, satisfactory pre-breakfast glucose results but persistently high pre-evening meal glucose in a patient on a single dose of basal insulin before bed may suggest a need to split the dose between before breakfast and in the evening.

Glucose monitoring is essential to achieving the goals within the Triangle of Diabetes Care, and the pros and cons of available methods are described below.

\section{The place of glucose monitoring within the Triangle of Diabetes Care \\ Self-monitoring of blood glucose (SMGB)}

SMBG technology has advanced greatly. Meters are now matchbox size and provide results in 5 seconds, often with 'smart' features such as internal memory to recall and graphically display previous blood glucose results and calculators to assist with insulin to carbohydrate dose adjustments. Nevertheless, SMBG remains unpopular, as it is messy, finger pricks are painful, testing is not discreet, and is perceived as a burdensome task. As a result, most patients do not measure their glucose sufficiently frequently for adequate assessment of their daily glucose profiles and glucose variability. Indeed many patients 'test' simply to confirm and then treat hypoglycaemia or to rule it out prior to driving or other important daily events.

Responding to the 'there and then' glucose values can lead to frequent snacking to reverse hypoglycaemic readings or taking extra rapid-acting insulin to treat a glucose peak ('chasing' the glucose). More motivated patients test and use the result together with the carbohydrate content of the meal to adjust insulin doses; regrettably, even the most motivated of 'testers' seldom use their results to build a profile of their daily glucose patterns which is essential to permit sensible tailoring of their insulin regimens. Indeed, even with frequent and diligent testing, the density of SMBG readings is often insufficient to build an adequate profile on which to base treatment change, particularly for readings at night.

\section{Continuous glucose monitoring (CGM)}

CGM has been available for more than 15 years. Recent devices provide real-time measurements of glucose levels, which can be displayed at 5-minute or 1-minute intervals. Alarms can be set to alert patients when the glucose levels are too low or too high (in practice many patients find these intrusive and switch them off). CGM is a major advance on SBGM, but devices remain relatively cumbersome, painful to insert, need finger-prick calibration, and often fail. More importantly, the density of data can bewilder patients and healthcare professionals, who may find the meter downloads from several days variable and difficult to interpret.

One important finding from CGM is a worrying frequency of

\section{Key messages}

- The management of glycaemia is usually based on measurement of $\mathrm{HbA}_{1 c}$, which tells us little about variability of blood glucose

- Glucose variability may be an important determinant of the risk of both hypoglycaemia and long-term diabetes complications

- The Triangle of Diabetes Care brings together the need for optimisation of the blood glucose level, avoidance of hypoglycaemia and reduction of glycaemic variability and is a useful concept within the management of diabetes

asymptomatic hypoglycaemia particularly at night. The recently introduced "Threshold Suspend" feature on some sensorassisted pump systems turns off insulin delivery when blood glucose falls below a preset threshold. ${ }^{20}$ This feature has been shown to significantly reduce nocturnal hypoglycaemia and is an important advance in care. ${ }^{17}$

\section{The place of ambulatory glucose profiles in assessing diabetes control}

CGM data are of potentially great value in understanding the facilitators and barriers to an individual's glycaemic control, as decribed above. However, the sheer weight of data produced, and their complexity, means that healthcare professionals may need additional support in using them effectively to optimise an insulin-based regimen. An expert panel of diabetes specialists, facilitated by the International Diabetes Center (IDC) and sponsored by the Helmsley Charitable Trust, met in 2012 to make recommendations for standardising the analysis and presentation of glucose monitoring data collection to aid clinical decision making in the management of diabetes.

The panel concluded that lack of a relatively simple and intuitive statistical and graphic visualisation of the glucose data from CGM downloads would represent a major contributor to the difficulties that clinicians and patients have in interpreting the data leading to uncertainty and reluctance to use CGM. This led to the endoresement of the concept of the 'ambulatory glucose profile' (AGP), originally developed by the IDC, as a statistical and visual representation of glycaemic exposure, variability, and hypoglycaemic risk. The AGP is presented in a series of dashboards which allow clinicians and patients to readily identify glucose patterns and address areas of highest clinical concern so that lifestyle and insulin therapy can be adjusted appropriately. The utility of the AGP is examined in more detail in the accompanying articles.

\section{Conclusions}

People with diabetes still struggle to improve overall diabetes control, despite major advances in diabetes technologies. In 
general, they remain fearful of hypoglycaemia, and find both SMBG and CGM of limited help in improving control and preventing hypoglycaemia. AGP is a unique means of presenting complex dense data gathered over an extended period from glucose monitoring systems in a straightforward, visual manner that supports healthcare professionals in making therapy decisions. Although AGP is a new technology, experience to date suggests that it could prove to be an important addition to diabetes care.

Conflict of interest Advisor to Abbott Diabetes, NovoNordisk UK, Lilly, SanofiDiabetes

Funding This supplement has been supported by a grant from Abbott Diabetes

\section{References}

1. Bliss M. The Discovery of Insulin. University of Chicago Press, p. 152.

2. Ainsberg A. Breakthrough - the story of Elizabeth Hughes and the making of a medical miracle. Available at https://www.idf.org/sites/ default/files/attachments/DV_56-SI2_Ainsberg.pdf (accessed December 2015).

3. Gebel E. The start of something good: the discovery of $\mathrm{HbA}(1 \mathrm{c})$ and the American Diabetes Association Samuel Rahbar Outstanding Discovery Award. Diabetes Care 2012;35:2429-31.

http://dx.doi.org/10.2337/dc12-1763

4. Inzucchi SE, Bergenstal RM, Buse JB, et al. Management of hyperglycaemia in type 2 diabetes, 2015: a patient-centred approach. Update to a position statement of the American Diabetes Association and the European Association for the Study of Diabetes. Diabetologia 2015; 58:429-42. http://dx.doi.org/10.1007/s00125-014-3460-0

5. Diabetes Control and Complications Trial Research Group. The effect of intensive treatment of diabetes on the development and progression of long-term complications in insulin-dependent diabetes mellitus. $N$ Engl J Med 1993;329:977-86. http://dx.doi.org/10.1056/NEJM199309303291401

6. UK Prospective Diabetes Study (UKPDS) Group. Intensive blood-glucose control with sulphonylureas or insulin compared with conventional treatment and risk of complications in patients with type 2 diabetes (UKPDS 33). Lancet 1998;352:837-53. http://dx.doi.org/10.1016/S0140-6736(98)07019-6

7. Holman RR, Paul SK, Bethel MA, et al. 10-year follow-up of intensive glucose control in type 2 diabetes. N Engl J Med 2008;359:1577-89.
http://dx.doi.org/10.1056/NEJMoa0806470

8. Nathan DM; DCCT/EDIC Research Group. The diabetes control and complications trial/epidemiology of diabetes interventions and complications study at 30 years: overview. Diabetes Care 2014;37:9-16. http://dx.doi.org/10.2337/dc13-2112

9. Cefalu WT, Ratner RE. The diabetes control and complications trial/epidemiology of diabetes interventions and complications study at 30 years: the "gift" that keeps on giving! Diabetes Care 2014;37:5-7. http://dx.doi.org/10.2337/dc13-2369

10. Frier BM, Jensen MM, Chubb BD. Hypoglycaemia in adults with insulintreated diabetes in the UK: self-reported frequency and effects. Diabet Med 2015 (advance online, http://dx.doi.org/10.1111/dme.12878).

11. Ceriello A, Kilpatrick ES. Glycemic variability: both sides of the story. Diabetes Care 2013;36 Suppl 2:S272-5. http://dx.doi.org/10.2337/dcS13-2030

12. Lachin JM, Genuth S, Nathan DM, Zinman B, Rutledge BN; DCCT/EDIC Research Group. The effect of glycemic exposure on the risk of microvascular complications in the diabetes control and complications trial-revisited. Diabetes 2008;57:995-1001. http://dx. doi.org/10.2337/db07-1618

13. Frontoni S, Di Bartolo P, Avogaro A, et al. Glucose variability: An emerging target for the treatment of diabetes mellitus. Diabetes Res Clin Pract 2013:102:86-95. http://dx. doi.org/10.1016/. diabres.2013.09.007

14. Ceriello A, Novials A, Ortega E, et al. Hyperglycemia following recovery from hypoglycemia worsens endothelial damage and thrombosis activation in type 1 diabetes and in healthy controls. Nutr Metab Cardiovasc Dis 2014;24:116-23. http://dx.doi.org/10.1016/j.numecd.2013.05.003

15. Almaatouq MA, Al-Arouj M, Amod A, et al. Barriers to the delivery of optimal antidiabetic therapy in the Middle East and Africa. Int J Clin Pract 2014;68:503-11. http://dx.doi.org/10.1111/ijcp.12342

16. Frier BM. Hypoglycaemia in diabetes mellitus: epidemiology and clinical implications. Nat Rev Endocrinol 2014;10:711-22. http://dx.doi.org/10.1038/nrendo.2014.170

17. Paty BW. The role of hypoglycemia in cardiovascular outcomes in diabetes. Can J Diabetes 2015;39 Suppl 5:S155-S159. http://dx.doi.org/10.1016/j.jcjd.2015.09.009

18. Cryer PE. Severe hypoglycemia predicts mortality in diabetes. Diabetes Care 2012;35:1814-6. http://dx.doi.org/10.2337/dc12-0749

19. Diabetes Control and Complications Trial Research Group. Hypoglycemia in the Diabetes Control and Complications Trial. Diabetes 1997:46:27186. http://dx.doi.org/10.2337/diab.46.2.271

20. Bergenstal RM, Klonoff DC, Garg SK, et al. Threshold-based insulinpump interruption for reduction of hypoglycemia. N Engl J Med 2013; 369:224-32. http://dx.doi.org/10.1056/NEJMoa1303576 